\title{
Post-processing Fibers for Sensing Applications
}

\author{
O. Frazão, M. S. Ferreira, R. M. André, S. O. Silva, M. B. Marques, José L. Santos \\ INESC Porto and Department of Physics and Astronomy, Faculty of Sciences, University of Porto, \\ Rua do Campo Alegre 687, 4150-179 Porto, Portugal
}

\begin{abstract}
A review in fiber post-processing for sensing applications is presented. The review is divided in three parts. Tapers devices, chemical etching for Fabry-Perot cavities and focused ion beam (FIB) as post-processing applied in optical fibers are considered. The most recent results as sensing elements are shown.

OCIS codes: (060.2370) Fiber optics sensors; (120.2230) Fabry-Perot; (230.4000) Microstructure fabrication.
\end{abstract}

\section{Introduction}

Post-processing of optical fibers provides a powerful mean of manipulating the characteristics of the guided light, opening up opportunities to create new sensors with high enhancement or with nanometers dimensions. One of the first devices subject to any post processing was the fiber taper. The tapers can be manufactured by etching or through a thermal stretching process. The first taper-based sensor was used as an optical refractometer. In 1984, Kumar et al. [1] presented a taper in multimode fiber as an optical intensity refractometer. In 1986, Boucouvalas et al. [2] studied the response of taper-based couplers to the refractive index of the external medium. Chemical etching is a simple method to make concavities in the tip of optical fibers forming Fabry-Perot (FP) cavities [3]. This method has been applied in different areas such as in the design of thermal probes sensors. The chemical etching was also applied in different topologies of fibers creating new geometries of Fabry-Perot. The advantages of using this technique are to reduce the dimensions of the cavities and to increase the sensitivity to the external medium. In the micro-fabrication process it is possible to obtain new enhancement of micro-sensors in optical fibers. Currently, two high technologies are explored called Focused Ion Beam (FIB) and femtosecond lasers. The FIB process [4] can create new design of microstructures such as micro-interferometers and micro-channels, in standard and microstructured optical fibers. The possibilities are immense and the research path will largely depend on the success in achieving more complicated structures.

In this presentation, we expect to present a review and new advances in post-processing in different sensing applications. Tapers for sensing applications based on standard microstructured fibers are explored as sensing element with high enhancement in physical parameter. Besides, Fabry-Perot cavities based on chemical etching are compared with the FIB manufacture process.

\section{Tapers sensors}

In 2006, it was developed a new concept to measure rotation angles based on a fiber-optic modal Mach-Zehnder interferometer by using a non-adiabatic taper cascaded with a long-period fiber grating (LPG) [5]. This configuration allowed obtaining information about the magnitude of the rotation angle from the measurement of the interference pattern visibility. It was also presented an in-fiber modal interferometer based on a non-adiabatic biconical fused taper that coupled light between the cladding and the core, combined with the Fresnel reflection at the fiber end. It was successfully demonstrated the use of this device as a fiber optic flowmeter [6] and inclinometer [7] sensor. Tapers combined with Bragg grating have also been explored. It was reported the analysis and experimental validation of the strain sensitivity dependences of a fiber Bragg grating (FBG) combined with fused tapers [8]. In 2011, it is presented the study over fiber structures based on the combination of abrupt tapers and FBGs [9]. It was demonstrated that an abrupt taper fabricated in the FBG region presented properties of a FabryPerot cavity; while an FBG written over fused tapers resulted in a phase-shifted FBG. In a different perspective, it was developed a fiber probe sensor for hydrogen detection that was based on a tapered-FBG [10].Multimode interferometer in taper is another topic very interesting for optical refractometer. It was demonstrated a configuration used a silica tube section spliced between two single-mode fibers, where in the splice regions tapers were made in order to allow the light to be guided in the silica tube while the core is formed by air [11]. Tapers in suspended core fiber presents also multimode interference and it was reported a study on strain sensitivity enhancement in suspended core fiber (SCF) tapers [12]. Tapers with different waist diameters were produced in SCF sections that were spliced between singlemode fibers (SMFs), allowed obtaining intermodal interference. More recently, André et al. [13] reported a high birefringence triangular optical nanowire by tapering SCFs down to core diameters below $1000 \mathrm{~nm}$. A nanowire was employed as a sensing element in a Sagnac interferometer for measuring 
temperature and a sensitivity of $-56.2 \mathrm{pm} / \mathrm{K}$ was attained. In Fig. 1 is presented several configurations of tapers in different types of fibers and combined with a FBG.
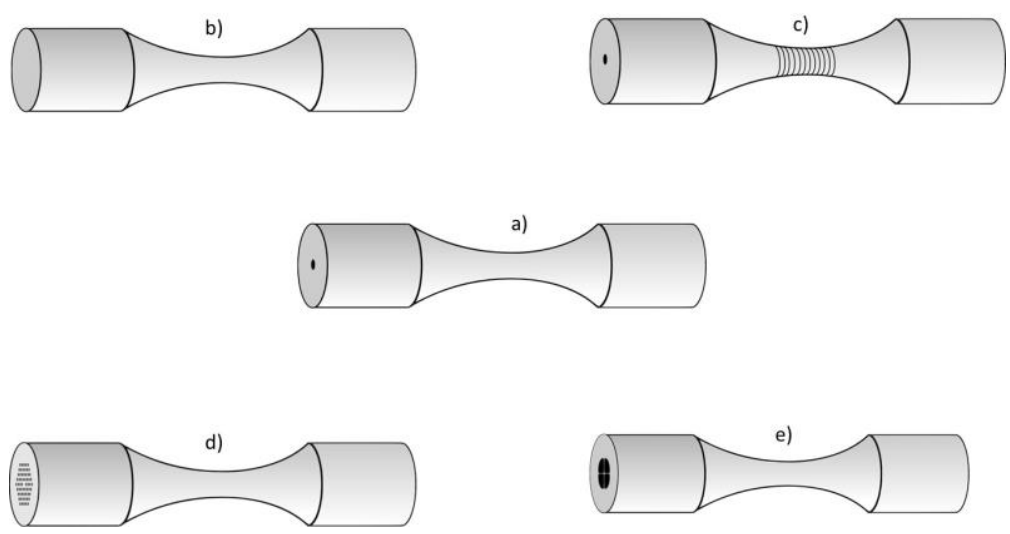

Fig. 1. Example of tapers: a) in single fiber; b) in multimode fiber; c) with a FBG; d) in PCF and e) SCF.

\section{Fabry-Perot based on Chemical etching}

The first work reporting the technique of using HF based chemical etching to produce Fabry-Perot cavities in SMF was published in 2006 [3]. This technique, offers low-cost and easy to manufacture fiber sensors that can be applied in several areas. These cavities are sensitive to physical, chemical and biological parameters. In 2012 two works were published on the chemical etching of graded index fibers $[14,15]$. The sensing heads were subjected to strain and temperature, where maximum sensitivities of $6.99 \mathrm{pm} / \mu \varepsilon$ and $0.95 \mathrm{pm} /{ }^{\circ} \mathrm{C}$ were respectively obtained. The post-processing of a double-clad optical fiber has also been studied $[16,17]$. In this situation, prior to etching there was no light propagation inside the core of the fiber. However, after the post-processing, the inner cladding was removed and the core begun to guide light. Two different configurations have been studied: with and without the incorporation of a diaphragm (see Fig. 2). In the first case, the sensors were subjected to temperature, whilst in the last the refractive index of water variation induced by temperature changes was studied. In the first sensing head configuration a sensitivity of $15.5 \mathrm{pm} /{ }^{\circ} \mathrm{C}$ was attained. Regarding the refractive index induced measurements in the second configuration, a sensitivity of $54.68 \mathrm{~nm} / \mathrm{RIU}$ was achieved.

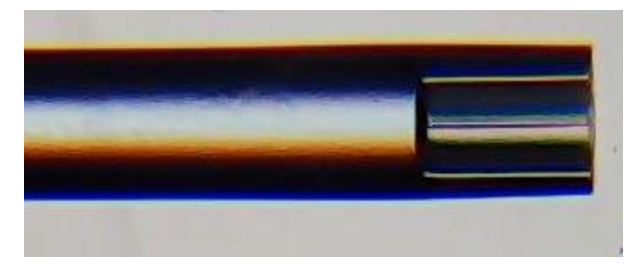

a)

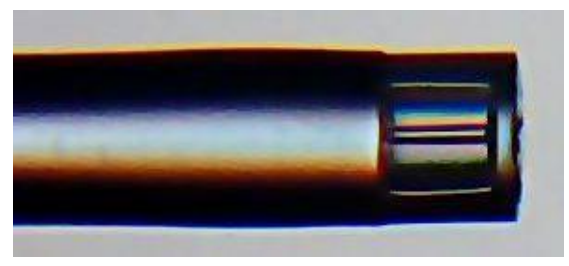

b)

Fig. 2. Photographs of the post-processed double-clad optical fiber sensor a) without diaphragm and b) with diaphragm.

\section{Microsensors using FIB technology}

FIB has been combined with optical fiber technology to create structures ranging from fiber Bragg gratings for refractive index [19] and temperature [20] sensing to more complex structures such as fiber-top cantilevers for very small displacement sensing [21]. Nanofiber cavities have been fabricated [22] by milling two FBGs on a nanofiber with FIB. FIB has also been used for the milling of channels in microstructured optical fibers, thus allowing for selective filling of these fibers [8]. Fabry-Perot (FP) cavities have also been fabricated using FIB. Most are created on tapered fiber tips in order to reduce the required FIB milling [9-11] to a minimum. One downside of FIB processing is the time it takes to mill large quantities of material. This is why, in the vast majority of the works involving FIB processing of optical fiber, another, faster technique is used to somehow reduce the amount of material to be milled. The most common method is tapering, either by creating nanofibers or tapered fiber tips. Recently, chemical etching of specifically designed fibers allows for the creation of microwires necessary to create a new structure embedded in the core fiber. Fabry-Perot structures were milled with FIB. One of the structures is a simple FP cavity created by milling an indentation in the microwire. See Fig. 3. 


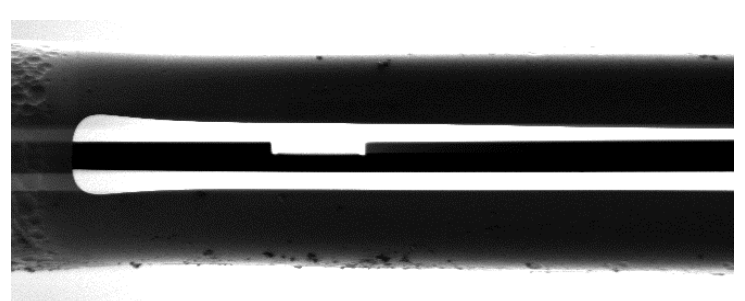

Fig. 3. Photographs of the post-processed FP cavity using FIB technology.

\section{Conclusions}

Summarizing, three mechanisms for post processing of fibers were described. Taper devices are at this moment a mature technology and in transfer to nanofibers. Chemical etching combined with FIB process has great potential in the near future especially in specific sensors with reduced size at the nanoscale.

\section{References}

[1] A. Kumar, T.V.B. Subrahmanyam, A.D. Sharma, K. Thyagarajan, B.P. Pal, and I.C. Goyal, Novel refractometer using a tapered optical fibre, Electronics Letters, 20, 534 (1984).

[2] A.C. Boucouvalas and G. Georgiou, External refractive-index response of tapered coaxial couplers, Optics Letters, 11, 257 (1986).

[3] V.R. Machavaram, R.A. Badcock, G.F. Fernando, Fabrication of intrinsic fibre Fabry-Perot sensors in silica fibres using hydrofluoric acid etching, Sensors and Actuators A: Physical, 138, 248-260, 2007.

[4] C. A. Volkert and A. M. Minor, "Focused Ion Beam Micromachining," Materials Research Society Bulletin, 32, 389-399, (2007).

[5] O. Frazão, R. Falate, J L Fabris, J. L. Santos, L. A. Ferreira, F. Araújo, Optical inclinometer based on a single long-period fiber grating combined with a fused taper, Optics Letters, 31, 2960-2962, (2006).

[6] O. Frazão, P. Caldas, F. M. Araújo, L. A. Ferreira, J. L. Santos, Optical flowmeter using a modal interferometer based on a single nonadiabatic fibre taper, Optics Letters, 32, 1974-1976, (2007).

[7] L. Amaral, O, Frazão, J. L. Santos, A. lobo, Fiber Optic Inclinometer Based on Taper Michelson Interferometer, IEEE Sensors Journal, 11, 1811-1814, (2011).

[8] O. Frazão, S. O. Silva, A. Guerreiro, J. L. Santos, L. A. Ferreira, F. M. Araújo, Strain Sensitivity Control of Fibre Bragg Grating Structures with Fused Tapers, Applied Optics, 46, 8578-8582, (2007).

[9] S. O.Silva, L. A. Ferreira, F. M. Araújo, J. L. Santos, O. Frazão, Fiber Bragg Grating Structures with Fused Tapers , Fiber and Integrated Optics, 30, 9-28, (2011).

[10] S. O. Silva, L. C. Coelho, J. Almeida, O. Frazão, J. L. Santos, F. X. Malcata, $\mathrm{H}_{2}$ Sensing Based on a Pd-Coated Tapered-FBG Fabricated by DUV Femtosecond Laser Technique, IEEE Photonics Technology Letters, 25, 401-403, (2013).

[11] Luís Carlos Coelho, J. Kobelke, K. Schuster, José Luís Santos, Orlando Frazão, Optical Refractometer Based on Multimode Interference in a Pure Silica Tube, Optical Engineering, 50, (2011).

[12] R. M. André, S. O. Silva, M, Becker, K. Schuster, M. Rothhardt, H. Bartelt, M. B. Marques, O. Frazão, Strain sensitivity enhancement in suspended core fiber tapers, Photonic Sensors, 1, 1-2, (2013).

[13] R. M. André, M. Becker, K. Schuster, M Rothhardt, H. Bartelt, M. B. Marques, O. Frazão, High Birefringence Triangular Optical Nanowire in Suspended-Core Fiber for Temperature Sensing, SPIE Nanophotonics, 7, 73088-73089, (2013).

[15] P. A. R. Tafulo, P. A. S. Jorge, J. L. Santos, F. M. Araújo, O. Frazão, "Intrinsic Fabry-Pérot Cavity Sensor based on Etched Multimode Graded Index fiber," IEEE Sensors Journal, 12, 8-12, (2012).

[16] P. A. R. Tafulo, P. A. S. Jorge, J. L. Santos, O. Frazão, "Fabry-Pérot cavities based on chemical etching for high temperature and strain measurement," Optics Communications, 285, 1159-1162, March 2012.

[17] M. S. Ferreira, J. Bierlich, K. Schuster, J. L. Santos, O. Frazão, Post-Processing of Fabry-Pérot Microcavity Tip Sensor, IEEE Photon. Tech. Lett., 25, 1593-1596, August 2013.

[18] M. S. Ferreira, J. Bierlich, S. Unger, K. Schuster, J. L. Santos, O. Frazão, Optical Phase Refractometer Based on Post-processed Interferometric Tip Sensors, IEEE Journal Lightwave Technology, (2014). Submitted.

[19] J.-L. Kou, S.-J. Qiu, F. Xu, Y.-Q. Lu, Y. Yuan, and G. Zhao, "Miniaturized Metal-Dielectric-Hybrid Fiber Tip Grating for Refractive Index Sensing," IEEE Photonics Technol. Lett. 23, 1712-1714 (2011).

[20] J. Kou, S. Qiu, F. Xu, and Y. Lu, "Demonstration of a compact temperature sensor based on first-order Bragg grating in a tapered fiber probe," Opt. Express, 19, 18452 (2011).

[21] D. Iannuzzi, K. Heeck, M. Slaman, S. de Man, J. H. Rector, H. Schreuders, J. W. Berenschot, V. J. Gadgil, R. G. P. Sanders, M. C. Elwenspoek, and S. Deladi, "Fibre-top cantilevers: design, fabrication and applications," Meas. Sci. Technol. 18, 3247-3252 (2007).

[22] K. P. Nayak, F. Le Kien, Y. Kawai, K. Hakuta, K. Nakajima, H. T. Miyazaki, and Y. Sugimoto, "Cavity formation on an optical nanofiber using focused ion beam milling technique," Opt. Express 19, 14040 (2011).

[23] F. Wang, W. Yuan, O. Hansen, and O. Bang, "Selective filling of photonic crystal fibers using focused ion beam milled microchannels," Opt. Express 19, 17585 (2011).

[24] J. Kou, J. Feng, Q. Wang, F. Xu, and Y. Lu, "Microfiber-probe-based ultrasmall interferometric sensor," Opt. Lett. 35, 2308 (2010).

[25] J. Kou, J. Feng, L. Ye, F. Xu, and Y. Lu, "Miniaturized fiber taper reflective interferometer for high temperature measurement," Opt. Express 18, 14245-14250 (2010). 